\title{
The Role of Healthcare Robotics in Providing Support to Older Adults: a Socio-ecological Perspective
}

\author{
George Mois ${ }^{1}$. Jenay M. Beer ${ }^{1,2}$ \\ Published online: 10 March 2020 \\ (C) Springer Science+Business Media, LLC, part of Springer Nature 2020
}

\begin{abstract}
Purpose of Review In this review, we provide an overview of how healthcare robotics can facilitate healthy aging, with an emphasis on physical, cognitive, and social supports. We next provide a synthesis of future challenges and considerations in the development and application of healthcare robots. We organize these considerations using a socio-ecological perspective and discuss considerations at the individual, care partner, community healthcare, and healthcare policy levels.

Recent Findings Older adults are the fastest growing segment of the US population. Age-related changes and challenges can present difficulties, for older adults want to age healthily and maintain independence. Technology, specifically healthcare robots, has potential to provide health supports to older adults. These supports span widely across the physical, cognitive, and social aspects of healthy aging.

Summary Our review suggests that while healthcare robotics has potential to revolutionize the way in which older adults manage their health, there are many challenges such as clinical effectiveness, technology acceptance, health informatics, and healthcare policy and ethics. Addressing these challenges at all levels of the healthcare system will help ensure that healthcare robotics promote healthy aging and are applied safely, effectively, and reliably.
\end{abstract}

Keywords Healthcare robotics $\cdot$ Healthy aging $\cdot$ Socio-ecological model $\cdot$ Robotics $\cdot$ Aging in place

\section{Introduction}

Older adults are a growing segment of the US population, with an estimated 83.7 million persons aged $65+$ by the year 2050 [1]. This growth can be attributed to advances in medicine and the aging of the baby boomer generation $[2,3]$. Majority of older adults and baby boomers wish to age in place [1], that is, they want to age in their own home and community [4-6]. However, many older adults experience barriers to aging in place [7], with unavailability of housing, inadequate or lack of healthcare resources, and scarcity of social supports perhaps the most pressing challenges. One approach to reducing these barriers and promoting aging in place, independence, and

This article is part of the Topical Collection on Home-Based Monitoring

Jenay M. Beer

Jenay.Beer@uga.edu

1 School of Social Work, University of Georgia, 279 Williams St, Athens, GA 30602, USA

2 Institute of Gerontology, University of Georgia, 102 Spear Road, Athens, GA 30606, USA healthy aging is the development and implementation of healthcare technology, such as healthcare robotics.

While there is no agreed upon definition of robots, they may be described as (semi) autonomous embodied computational systems that can sense the environment and can then act on the environment to achieve some goals [8]. Robots are in early stages of commercial development; however, with computational and mechanical advancements, robots will increasingly be adopted and implemented in the homes of older adults to promote healthy aging [9-12].

In this review, we will first provide an overview of how robots can provide physical, cognitive, and social support to older adults. We acknowledge that a number of in-depth reviews are already published [13-15]. While these previous reviews provide an in-depth synopsis of the state of the art of the healthcare robotics field, our goal is to provide an overview of healthcare robotics to support healthy aging as a contextual reference. We aim to focus on the future challenges of healthcare robotics and how such technology can support healthy aging and our healthcare system. Therefore, we next categorize future humanrobot interaction (HRI) considerations and challenges at the individual, interpersonal, community, and societal levels via a 
socio-ecological inspired framework. The novelty of this review is this multilevel framework to be used as a useful organizational structure to consider the future of integration of robotics at multiple levels of older adult healthcare. We will close this review with acknowledging gaps in the literature and considerations for future research in healthcare robotics.

\section{Overview of Healthcare Robotics to Support Healthy Aging}

Aging in place is attributed to many health benefit [16, 17] promotion of independence and dignity. These are important factors in healthy aging. Healthy aging can be defined as "the development and maintenance of optimal physical, mental, and social well-being and function in older adults [ ... achieved when communities are safe, promote health and well-being, and use health services and community programs to prevent or minimize disease" [18]. This definition is important because it highlights the physical, mental (cognitive), and social aspects of healthy aging - all areas in which healthcare robotics has potential to support. Healthcare robotics is an umbrella term that describes a number of robot classifications; social companion robots, assistive robots, tele-robots, telepresence robots, and socially assistive robots (SARs) being a few common terms used in the literature. These types of robots serve both independent and shared functions; however, we will focus less on the classification of robot (for overview of healthcare robot classifications, see [19]) and instead focus on how healthcare robots, broadly defined, can provide physical, cognitive, and social supports for older adults.

Majority of healthcare robots have been developed to assist older adults with physical tasks, such as activities of daily living (ADLs) or instrumental activities of daily living (IADLs) [20]. Typically, commercially available robots that assist with physical tasks are specialty robots - that is, they perform a single task. For example, assistance with home cleaning is a task many older adults state that they feel comfortable with a robot assisting with. The Roomba, to date, is perhaps the most popular specialized home cleaning robot assistant that is commercially available [21]. This robot assists with vacuuming, using sensors and artificial intelligence to learn an environment. Specialized assistance with home cleaning tasks, such as vacuuming, can remove the burden of having to carry out tasks that can be physically demanding. Roomba, and other robots like it, can help older adults maintain their home and age in place successfully. In fact, older adults have expressed the most interest in robots which could assist in performing IADLs [22-24], such as chores (e.g., housework), manipulating objects (e.g., lifting heavy objects), and information management tasks (e.g., reminding, monitoring a person, and transmitting information for help if the person falls).

Robots such as Nursebot can help provide auto reminders for older adults about events, schedules, and even medication management [25]. Pearl, Cafero, and iRobiQ were also specifically designed to assist older adults with medication and calendar reminders $[26,27]$. However, data from a series of qualitative studies [22-24] has shown that older adults were selective about task preference, even within a healthcare category. For instance, older adults prefer robot assistance with medication reminders and delivery (e.g., a robot can bring a bill bottle to an older adult), whereas they preferred human assistance medication decision-making [23]. Therefore, for even highly related healthcare tasks, such as medication management, older adults were discriminating.

Despite older adults' acceptance of robots assisting with reminders, the actual development and implementation of robotic cognitive support are complex and under studied [28]. Within the area of neuropsychology, healthcare robotics have been applied as psychometric tools for cognitive assessment to help detect cognitive impairment in older adults through social interactions $[29,30]$. Robots have also been shown to be generally useful in other cognitive-related areas such as cognitive education, rehabilitation, and skill training [28, 31-33]. The application of robots to provide individuals with health training, cognitive training, and health service delivery is a growing area of HRI, but additional research is needed to determine the feasibility, develop design specifications, and determine how older adults react to a robotic cognitive trainer.

In contrast, older adults have expressed a preference for human assistance (not robot assistance) for most physical ADL tasks related to personal care (e.g., bathing, grooming, meal prep) [22, 23]. Despite this, regarding assistance with physical activities of daily living (ADLs), healthcare robots that provide mobility supports are increasingly research and developed - specifically mobility devices in the form of robotic wheelchairs or walkers [24] that can support older in avoiding obstacles and aid with navigation. Currently, it is uncommon to find commercially available robots that provide support with other physical activities such as feeding, bathing, toileting, and dressing. Development of such robots requires considerable computational advancement to operate safely in close proximity to a persons' physical body [34] and take into account a variety of user needs, capabilities, and preferences [35]. However, the development of robots to address these activities could benefit a large number of older adults [34], that is, if older adults are willing to adopt them [22, 23].

Finally, healthcare robots hold the potential to help older adults age healthily with a variety of social supports. Social robots have been researched and developed to operate as a number of social companion roles, such as partners, peers, or assistants; this will allow them to exhibit flexibility and adaptability [36] to support social interaction with a wide range of older adults. Social robots, such as companion robots, can help older adults remain engaged and assist with social interaction [37, 38], as well as improve psychological status and overall well-being [39]. The most widely researched and 
available companion robot is Paro, a robotic seal. Paro, as well as other companion robots, has been shown to reduce stress and encourage socialization, improving older adults' quality of life [34, 40-43]. Furthermore, companion robots have contributed to a decrease loneliness and increased emotional engagement among older adults [44]. Moreover, healthcare robotics can help support interaction between users. Telepresence robots are defined as systems that provide twoway audio and video, on a mobile base (sometimes referred to as tele-conferencing on wheels). Telepresence robots can enable older adults to connect to their remotely located family, friends, and health providers [45, 46].

In summary, the literature suggest that healthcare robotic assistance may be one way for older adults to select, optimize, or compensate for age-related changes and challenges. However, it is important to note that for physical, cognitive, and social assistance, the needs of an older adult will change over time. Therefore, healthcare robots will need to adapt with the individual, his or her needs, and the environment. Moreover, older adults could benefit from earlier adoption of robots into their lives, because age-related declines (e.g., physical, perceptual, cognitive age-related changes) may make learning how to use and adopt a robot more difficult.

\section{A Socio-ecological Framework for Healthcare Robotics and Aging}

As outlined above, a considerable amount of research has been geared toward understanding the role of healthcare robots in the provision of services to older adults. These services can play an essential role in supporting healthy aging, maintaining independence, and endorsing dignity. However, for healthcare robotics to provide support in healthy aging, we need to understand the future challenges and considerations for robot implementation at various healthcare levels. To accomplish this, we propose a framework that considers the role of robotics across the socio-ecological levels of healthcare. Each of these levels poses specific challenges and considerations pertaining to the design, development, and implementation of healthcare robots. Furthermore, the framework presents an organizational structure at four distinct nested levels, which include the individual, care partners, community healthcare, and the healthcare system (State/Federal) (see Fig. 1).

\section{Levels 1 and 2: Individual (Older Adult User) and Care Partners (Family and Friends)}

The first two socio-ecological levels, the individual and care partners, share common HRI considerations as the interactions between human and technology are often situated in close proximity between that of the one receiving support (user) and the individual being assisted in providing support (care partner). Future challenges and considerations span across three key categories; these categories include user demographics, acceptance, technology competency, literacy, and clinical effectiveness.

\section{User Demographics}

The consideration of user demographics plays an essential role in developing healthcare robots. With a growing diverse older adult population, it is crucial that robots are equipped with the hardware and software needed to meet the various individual needs of older adults. These considerations include gender, age, race, ethnicity, educational attainment, and socioeconomic status. The effectiveness of healthcare robotics in supporting healthy aging is dependent on these demographics, which are identified as moderators of technology acceptance [47, 48]. Demographic considerations need to be extended to the older adults' care partner(s). For example, the age of care partner can vary significantly as a care partner may include a family member, a friend, or a formal caregiver. Therefore, familiarity and comfort level with technology use, including healthcare robotics, may vary. Furthermore, often discussed in the literature is the high cost often associated with robots $[49,50,51 \bullet]$. These barriers hold weight in ensuring that all older adults and care partners are able to access and afford healthcare robots. This is particularly important as healthcare robots may have the potential to improve access to quality healthcare for individuals who may otherwise have limited or no access $[52 \bullet \bullet$.

\section{Acceptance, Technology Competency, Literacy}

Healthcare robots will be effective only if an older adult is willing to accept and adopt the technology into their home. The Unified Theory of Acceptance is a theoretical model that includes perceived usefulness, perceived ease of use, social influences, and facilitating conditions as key variables that predict technology adoption [53]. However, the older adult and care partners' technological competency and literary are important considerations throughout the adoption and post adoption phases. For example, a user whom has adopted a healthcare robot but lacks comprehensive understanding of the capabilities and limitations of the technology, and does not fully understand how to use it, may experience negative relationship and satisfaction with the healthcare robot. Robot acceptance has been well-studied [54]; however, there are still remaining questions regarding why older 


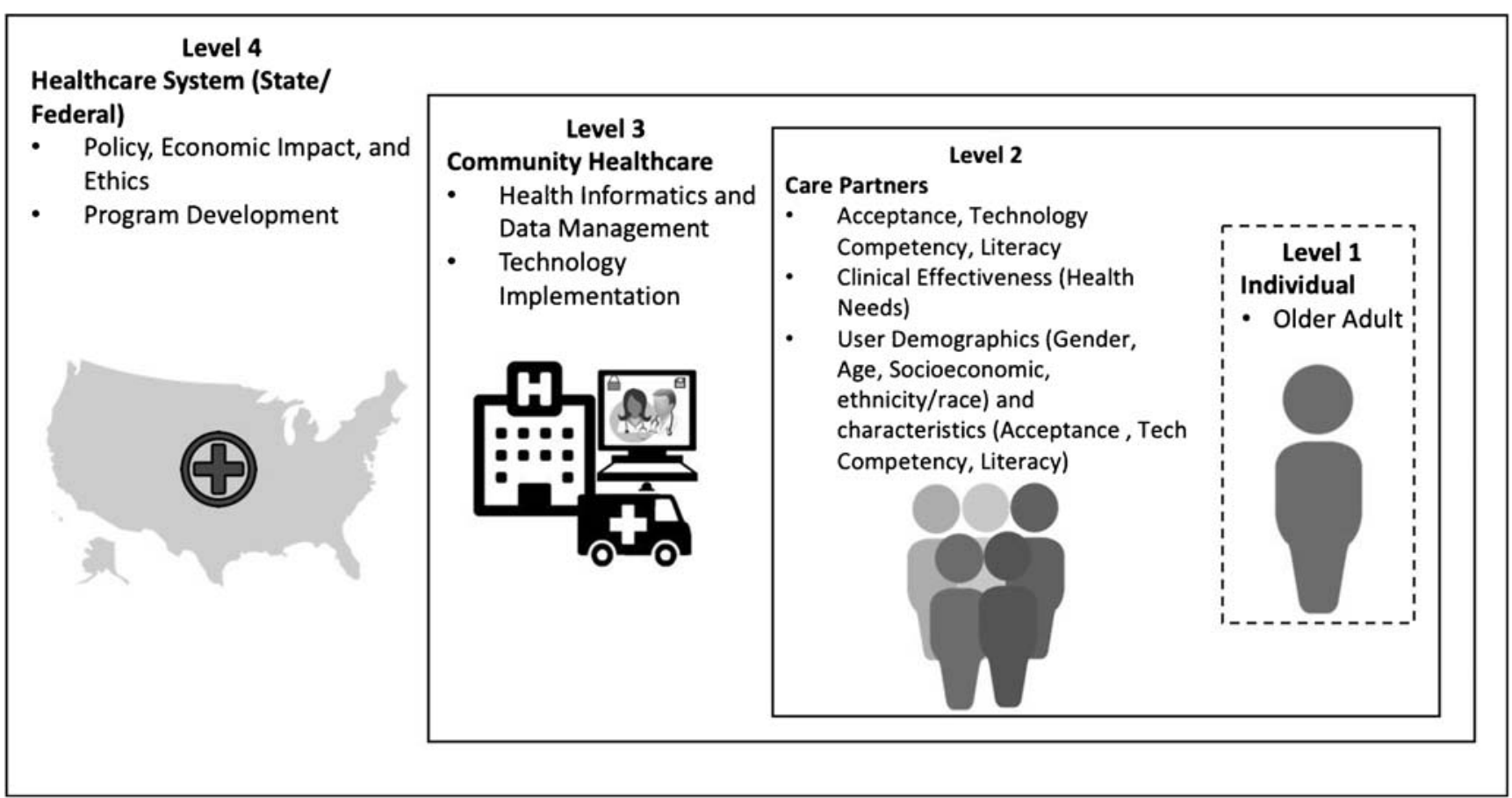

Fig. 1 Socio-ecological framework for health robotics

adults hold certain attitudes toward robots and what exactly drives their decision.

\section{Clinical Effectiveness (Health Needs)}

Clinical effectiveness is a prerequisite in ensuring that healthcare robotics are capable of providing the support required by older adults $[50,55]$. However, clinical effectiveness will differ from one older adult to another. Older adults can have a diverse set of needs that need to be met to ensure clinical effectiveness. For example, the set of needs of an older adult experiencing early onset of mild cognitive impairment but is socially connected and is in good physical health are going to be different than that of an older adult whom is experiencing multiple chronic conditions, reports being lonely, and has been diagnosed with severe mild cognitive impairment [56]. Furthermore, health is dynamic; sudden changes in health needs may require adaptation and adjustments by a healthcare robot. Thus, robots need to not only be customized but also adjust the level of care, depending on the everchanging needs of the older adult.

Setting clear guidelines and ensuring the clinical effectiveness of a healthcare robot before implantation in residential and institutional settings can affect long-term use and the robots' proficiency in providing the proper support for both the user and care partners. Considering existing clinical effectiveness benchmarks in any research pertaining to healthcare robotics is of major importance in adequately meeting the health needs of older adults [57].

\section{Level 3: Community Healthcare}

The third socio-ecological level of the proposed framework is community healthcare. Community healthcare encompasses the spectrum of healthcare providers, which includes both professionals and healthcare institutions. These considerations span across two key categories; these categories include health informatics and data management and technology implementation.

\section{Health Informatics and Data Management}

The term health informatics is used to describe the process of storage, retrieval, and processing of health data to create a collaborative approach towards healthcare provision [58]. Healthcare robots serve as the technology medium through which user data is collected and shared with a community healthcare provider. Robotic systems may utilize a series of sensors to measure the user's vital signs, track movements, and promote social engagement $[47,59]$. Health informatics, in the context of healthcare robotics literature, refers to the data collected and utilized to help facilitate more effective and efficient healthcare interventions. By leveraging the power of health informatics, data can be utilized and leveraged by care practitioners to enhance communication, educate clients, promote safety, and enhance patient care. Furthermore, the compilation of this data can inform research pertaining to healthcare robotics and help provide artificial intelligence systems with vast case examples on which information may be drawn from $[58,60,61]$. However, the current literature points 
to some key concerns pertaining to healthcare robots' data collection, synthesis, and analysis - these being privacy, safety, reliability, and usability [52••]. For example, to promote privacy, it is important that the older adult has control over what health information is being collected and what is being done with that information (e.g., with whom, when, and how it shared).

\section{Technology Implementation}

Healthcare communities can extend and enhance their services though healthcare robots to provide older adults and their care partners a set of tools that can support healthy aging [62]. Most older adults in the USA report that their preferred living arrangement is to age in place $[6,63,64]$. The implementation of healthcare robots across various living arrangements requires a dynamic set of functions and technical specifications needed to ensure effectiveness and suitability [62, 65]. Implementation of technology in older adults' homes can be challenging, as not all older adults have access to highspeed networking and many (most) older adult homes are not ADA compliant (American Disabilities Act). For example, a home-based healthcare robot may need to navigate stairs, multiple types of flooring, and dynamic obstacles (e.g., furniture, pets) to operate effectively, whereas a robot deployed in an institutional setting (e.g., assisted living or retirement community) will operate in a more controlled setting with wider doorways, ADA accessible floors (e.g., elevators and ramps), and IT support. Therefore, the computational and engineering requirements for healthcare robots to be implemented in diverse settings (home vs. institutional care) will vary considerably. Not only will the robot need to adapt to the environment but also adapt to the individual needs of the older adult and care partners. Therefore, high levels of robot functionality and artificial intelligence will be required to support healthy aging.

The alternative to aging in place, for many older adults, has become institutionalized care. Institutionalized care provides older adults with well-designed facilities and a wide variety of amenities [66]. However, institutionalized care living arrangements can range depending on the level of care the older adult requires, ranging from independent living to a high level of care assisted living. For example, an individual living in an independent living facility, who is experiencing some difficulty with ADLs, has a chronic heart condition, and is diagnosed with early-stage Alzheimer's disease, may require robot assistance for multiple tasks. These tasks may include medication dispensation, vitals tracking, cognitive training, therapy, and assistance with ADL [52••, 67-69]. The involvement of healthcare robotics within the context of healthcare communities presents new opportunities toward improving the provision and access to health services.

\section{Level 4: Healthcare System (State/Federal)}

The fourth socio-ecological level of the proposed framework is the healthcare system. The healthcare system encompasses the spectrum of governing bodies at both the state and federal levels, which help influence and set the policies for healthcare provision and access. These considerations span across two key categories; these categories include policy, economic impact, and ethics and program development.

\section{Policy, Economic Impact, and Ethics}

As the number of older adults continues to grow, the resources required to support healthy aging are also growing. This challenge is emerging due to economic strain, but also due to a shortage of qualified healthcare workers. The emergence of healthcare robotics presents a promising potential in creating significant cost savings and improving the provision of medical services. Access to these technological resources, such as robotics, is often dependent on the support of State and Federal policies which can help promote and improve aspects for all members of society. However, the role of policy needs to also account for the implication of healthcare robots' effect on the overall well-being of society and the entirety of the healthcare system [70].

The emergence of healthcare robots within the healthcare system holds many potential benefits toward improving access and affordability of health services. As a new emerging format for communication, service delivery, and patient interaction, discussions pertaining to ethical principles around the production, implementation, and use of healthcare robots are of tremendous relevance. Some of the key ethical concerns identified across the literature include implications for the labor field [71], quality of care [72], user autonomy [73], moral agency, responsibility [51•], deception [74], privacy, and safety [51•]. Ethical principles are needed to help structure practitioners' and providers' understanding of how and when healthcare robots might be used $[75,76]$. For example, the replacement of medical providers by healthcare robots may create an elimination of a human element in healthcare service provision [51•]. Furthermore, the increased involvement of robots in the provision of care may also affect individuals' moral agency and autonomy. Policies should aim to help regulate and ensure the safety and efficacy of interventions carried out by healthcare robots. Furthermore, policies should help safeguard and regulate data collection, data sharing, security, and privacy of the individual. The outcome goal of such standards is to promote independence, dignity, and user autonomy [23, 41, 42, 77, 78].

At a system level, well-established ethical standards can carefully monitor the applications and capabilities of robots to address concerns pertaining to safety and privacy $[25,39$, $40,68,69]$. The goal of a well-established ethical standards set 
out by healthcare systems at both a federal and state level is to help protect the users and ensure that their needs and wellbeing are prioritized in the implementation of healthcare robotics. It is important to note that the emergence of ethical standards needs to be sensitive to rapid innovation, which often occurs in this field. Therefore, ethical standards need to be flexible and adjusted based on current technological innovations.

\section{Program Development}

The healthcare system holds an important role in the development of educational tools and programs designed to provide both users and service providers the training to implement and utilize healthcare robotics effectively [70]. Developing a structured system-wide approach can help raise awareness and improve access to technological resources [79]. Ensuring program development includes and addresses that the importance of healthcare robotics education can have tremendous implications for clinical effectiveness in the healthcare community and at the user and care partner level. Furthermore, the utilization of such a system can help generate a more accurate understanding of user and service provider's needs, thus informing the development of healthcare robotics. Program development involving the use of healthcare robotics need to be informed by system-wide ethical standard and aim to address implication for various professional code of ethics of healthcare provider personal [51•]. The goal of programs should be centered on the promotion of healthy aging and ensuring the wellbeing of older adults.

\section{Conclusion}

In summary, this review provides an overview of healthcare robotics potential to support older adults in healthy aging. Specifically, we highlight the potential for robots to assist with physical, cognitive, and social supports. To date, much of the robotics literature focuses on the research, development, and implementation of healthcare robots to assist with physical tasks, such as IADLs [34, 80, 81]. Older adults are somewhat less accepting of robots assisting with personal care tasks (e.g., some ADLs such as grooming and dressing); however, if robots can safely and reliably be developed to assist with these tasks, they have potential to provide support to many older adults with age-related declines [13, 82, 83]. Healthcare robots also hold much potential in providing social supports particularly telepresence robotics that can facilitate social connection with remote family, friends, and healthcare providers [84-87].

Additionally, we provided a review of future challenges and considerations in implementing healthcare robotics. We utilize a socio-ecological perspective to organize and describe these challenges along the individual, care partner, community healthcare, and the Federal/State healthcare system. This organizational structure is useful, although it is important to note that this is nested system and many of the issues discussed in this paper (e.g., technology acceptance, ethics, clinical effectiveness) span across multiple levels. While our review is not exhaustive, we believe that our synthesis of the literature provides a roadmap for future research and technology development - with a plethora of exciting considerations and challenges to address. Healthcare robotics holds much promise to support older adults in healthy aging. Thus, consideration of the technology's implications at all levels of the healthcare system is a crucial step forward in making healthcare robotics a universally accepted technology in older adults' daily care.

\section{Compliance with Ethical Standards}

Conflict of Interest The authors declare that they have no conflict of interest.

Human and Animal Rights This article does not contain any studies with human or animal subjects performed by any of the authors.

\section{References}

Papers of particular interest, published recently, have been highlighted as:

- Of importance

•. Of major importance

1. Bardic A. Time to rethink the boomers? Buying power of the 50plus crowd still packs a punch. Convenience Store News 2015;136.

2. Mellor JM, Rehr H. Baby boomers: can my eighties be like my fifties? New York: Springer Publishing Company; 2005. 208 p. 9.

3. Kleyman P. The age of anti-aging: media hype and the myth of the ageless baby boomer. Generations. 2017;41:41-7.

4. Blanchard J. Aging in Community: Communitarian Alternative to Aging in Place, Alone [Internet]. 2013 [cited 2020 Jan 4]. Available from: https://www.ingentaconnect.com/content/asag/gen/2013/ 00000037/00000004/art00002. Accessed 4 Jan 2020.

5. Dye CJ, Willoughby DF, Battisto DG. Advice from rural elders: what it takes to age in place. Educ Gerontol. 2011;37:74-93.

6. Kim K, Gollamudi SS, Steinhubl S. Digital technology to enable aging in place. Exp Gerontol. 2017;88:25-31.

7. Black K, Dobbs D, Young TL. Aging in community: mobilizing a new paradigm of older adults as a core social resource. J Appl Gerontol. 2015;34:219-43.

8. Matarić MJ. The robotics primer. Cambridge Mass.: MIT Press; 2007.

9. Beer JM, Mitzner TL, Stuck RE, Rogers WA. Design considerations for technology interventions to support social and physical wellness for older adults with disability. International Journal of Automation and Smart Technology [Internet]. 2015;(4):249.

10. Forlizzi J, Disalvo C, Gemperle F. Assistive robotics and an ecology of elders living independently in their homes. Human Compu. 2004;19:25-59. 
11. Pedersen I, Reid S, Aspevig K. Developing social robots for aging populations: a literature review of recent academic sources. Sociol Compass. 2018:e12585.

12. Smarr C-A, Prakash A, Beer JM, Mitzner TL, Kemp CC, Rogers WA. Older adults' preferences for and acceptance of robot assistance for everyday living tasks. Proc Hum Factors Ergon Soc Annu Meet. 2012;56:153-7.

13. Broadbent E, Stafford R, MacDonald B. Acceptance of healthcare robots for the older population: review and future directions. Int $J$ of Soc Robotics. 2009;1:319.

14. Moerman CJ, van der Heide L, Heerink M. Social robots to support children's well-being under medical treatment: a systematic stateof-the-art review. J Child Health Care. 2019;23:596-612.

15. Zhao J-X, Li C, Ren H, Hao M, Zhang L-C, Tang P-F. Evolution and current applications of robot-assisted fracture reduction: a comprehensive review. Ann Biomed Eng. 2020;48:203.

16. Black K. Health and aging-in-place: implications for community practice. J Community Pract. 2008;16:79-95.

17. Cutchin MP. The process of mediated aging-in-place: a theoretically and empirically based model. Soc Sci Med. 2003;1077.

18. Lang JE, Anderson L, James L, Sharkey J, Belansky E, Bryant L, et al. The prevention research centers healthy aging research network. Prev Chronic Dis. 2005;3:3.

19. Mois G, Beer JM. Chapter 3 - Robotics to support aging in place. In: Pak R, de Visser EJ, Rovira E, editors. Living with robots [Internet]. Academic Press; 2020. p. 49-74.

20. Feil-Seifer D, Skinner K, Matarić M. Benchmarks for evaluating socially assistive robotics. Interact Stud. 2007;8:423-39.

21. Roomba Robot Vacuum Cleaners | iRobot [Internet]. [cited 2020 Jan 5]. Available from: https://www.irobot.co.uk/roomba. Accessed 5 Jan 2020.

22. Beer JM, Prakash A, Smarr C-A, Chen TL, Hawkins K, Nguyen H, et al. Older users' acceptance of an assistive robot: attitudinal changes following brief exposure. Gerontechnology. 2017;16:21-36.

23. Smarr C-A, Mitzner TL, Beer JM, Prakash A, Chen TL, Kemp CC, et al. Domestic robots for older adults: attitudes, preferences, and potential. Int J Soc Robot. 2014;6:229-47.

24. Smarr C-A, Fausset CB, Rogers WA. Understanding the potential for robot assistance for older adults in the home environment. [Internet]. Atlanta, GA: Georgia Institute of Technology; 2011.

25. Matthews JT. The Nursebot project: developing a personal robotic assistant for frail older adults in the community. Home Health Care Manag Pract. 2002;14:403-5.

26. Pollack M, Brown L, Colbry D, Orosz C, Peintner B, Ramakrishnan S, et al. Pearl: A Mobile Robotic Assistant for the Elderly. 2002;

27. Rebok GW, Carlson MC, Langbaum JBS. Training and maintaining memory abilities in healthy older adults: traditional and novel approaches. J Gerontol B Psychol Sci Soc Sci. 2007;62(1):53-61.

28. Tsiakas K, Abellanoza C. Towards designing a socially assistive robot for adaptive and personalized cognitive training. 2017.

29. Varrasi S, Di Nuovo S, Conti D, Di Nuovo A. Social robots as psychometric tools for cognitive assessment: a pilot test. Human Friendly Robotics. Springer; 2019. p. 99-112.

30. Jøranson N, Pedersen I, Rokstad AMM, Ihlebæk C. Effects on symptoms of agitation and depression in persons with dementia participating in robot-assisted activity: a cluster-randomized controlled trial. J Am Med Dir Assoc. 2015;16:867-73.

31. Campa R. The rise of social robots: a review of the recent literature. J Evol Technol. 2016;26.

32. Eftring H, Frennert S. Designing a social and assistive robot for seniors. Z Gerontol Geriat. 2016;49:274-81.

33. Quick T, Dautenhahn kerstin. Making embodiment measurable. Proceedings of ' 4 Fachtagung der Gesellschaft für Kognitionswissenschaft' Bielefeld, Germany http://supergoodtech. com/tomquick/phd/kogwis/webtext.html. 1999.
34. Mitzner TL, Chen TL, Kemp CC, Rogers WA. Identifying the potential for robotics to assist older adults in different living environments. Int J Soc Robot. 2014;6:213-27.

35. Canal G, Alenyà G, Torras C. A taxonomy of preferences for physically assistive robots. 2017 26th IEEE International Symposium on Robot and Human Interactive Communication (RO-MAN). 2017. p. 292-7.

36. Fong T, Nourbakhsh I, Dautenhahn K. A survey of socially interactive robots. Robot Auton Syst. 2003;42:143-66.

37. Huschilt J, Clune L. The use of socially assistive robots for dementia care. J Gerontol Nurs. 2012;38:15-9.

38. Pineau J, Montemerlo M, Pollack M, Roy N, Thrun S. Towards robotic assistants in nursing homes: challenges and results. Robot Auton Syst. 2003;42:271-81.

39. Reiser U, Jacobs T, Arbeiter G, Parlitz C, Dautenhahn K. Care-Obot ${ }^{\circledR} 3$ - vision of a robot Butler. In: Trappl R, editor. Your virtual Butler: the making-of [internet]. Berlin: Springer; 2013 [cited 2020 Jan 5]. p. 97-116. https://doi.org/10.1007/978-3-642-37346-6 9.

40. Kidd CD, Taggart W, Turkle S. A sociable robot to encourage social interaction among the elderly. Proceedings 2006 IEEE International Conference on Robotics and Automation, 2006 ICRA 2006 [Internet]. Orlando, FL, USA: IEEE; 2006 [cited 2020 Jan 5]. p. 3972-6. Available from: http://ieeexplore.ieee.org/document/ 1642311/. Accessed 5 Jan 2020.

41. Lane GW, Noronha D, Rivera A, Craig K, Yee C, Mills B, et al. Effectiveness of a social robot, "Paro," in a VA long-term care setting. Psychol Serv. 2016;13:292-9.

42. McGlynn SA, Kemple S, Mitzner TL, King C-HA, Rogers WA. Understanding the potential of PARO for healthy older adults. Int J Hum Comput Stud. 2017;100:33-47.

43. Wada K, Shibata T, Saito T, Sakamoto K, Tanie K. Psychological and social effects of one year robot assisted activity on elderly people at a health service facility for the aged. Proceedings of the 2005 IEEE International Conference on Robotics and Automation. 2005. p. 2785-90.

44. Robinson H, Broadbent E, MacDonald B. Group sessions with Paro in a nursing home: structure, observations and interviews. Australas J Ageing. 2016;35:106-12.

45. Beer JM, Takayama L. Mobile remote presence systems for older adults: acceptance, benefits, and concerns. Proceedings of the 6 th international conference on Human-robot interaction - HRI '11 [Internet]. Lausanne, Switzerland: ACM Press; 2011 [cited 2019 Jan 9]. p. 19. Available from: http://portal.acm.org/citation.cfm? doid=1957656.1957665. Accessed 4 Jan 2020.

46. Kristoffersson A, Coradeschi S, Loutfi A. A review of mobile robotic telepresence. Adv Hum Comput Interact. 2013;2013:1-17.

47. Beuscher LM, Fan J, Sarkar N, Dietrich MS, Newhouse PA, Miller KF, et al. Socially assistive robots. J Gerontol Nurs. 2017;43:35-43.

48. Rojas-Méndez JI, Parasuraman A, Papadopoulos N. Demographics, attitudes, and technology readiness: a crosscultural analysis and model validation. Mark Intell Plan. 2017;35:18-39.

49. Jones SS, Koppel R, Ridgely MS, Palen TE, Wu S-Y, Harrison MI. Guide to reducing unintended consequences of electronic health records: [Internet]. 2011 [cited 2019 Dec 30]. Available from: https:// www.rand.org/pubs/external_publications/EP201100261.html. Accessed 30 Dec 2019.

50. Kneuertz PJ, Singer E, D'Souza DM, Abdel-Rasoul M, MoffattBruce SD, Merritt RE. Hospital cost and clinical effectiveness of robotic-assisted versus video-assisted thoracoscopic and open lobectomy: a propensity score-weighted comparison. J Thorac Cardiovasc Surg. 2019;157:2018-2026.e2.

51. Stahl BC, Coeckelbergh M. Ethics of healthcare robotics: towards responsible research and innovation. Robot Auton Syst. 2016;86: 152-61. This article highlight important concerns and ethical considerations pertaining to healthcare robotics research. 
52.• Simshaw D, Terry N, Hauser K, Cummings M. Regulating healthcare robots: maximizing opportunities while minimizing risks [Internet]. Rochester: Social Science Research Network; 2016. Report No.: ID 2739462. Available from: https://papers.ssrn.com/abstract=2739462 Accessed 30 Dec 2019. This article highlight the role of healthcare robotics, identifies current and emerging innovations, and examines the regulatory framework for these technologies.

53. Venkatesh V, Morris MG, Davis GB, Davis FD. User acceptance of information technology: toward a unified view. MIS Q. 2003;27: 425-78.

54. Heerink M, Kröse B, Evers V, Wielinga B. Assessing acceptance of assistive social agent technology by older adults: the Almere model. Int J of Soc Robotics. 2010;2:361-75.

55. Park DA, Yun JE, Kim SW, Lee SH. Surgical and clinical safety and effectiveness of robot-assisted laparoscopic hysterectomy compared to conventional laparoscopy and laparotomy for cervical cancer: a systematic review and meta-analysis. Eur J Surg Oncol. 2017;43:994-1002.

56. Bucciarelli A. Alzheimer's disease [internet]. Bloomfield: Mercury Learning \& Information; 2015. Available from: http://proxyremote.galib.uga.edu/login?url=http://search.ebscohost.com/login. aspx?direct=true $\& \mathrm{db}=\mathrm{e} 000 \mathrm{xna} \& \mathrm{AN}=1809102 \&$ site $=$ eds-live. Accessed 29 Dec 2019.

57. Begum M, Serna RW, Yanco HA. Are robots ready to deliver autism interventions? A comprehensive review. Int J of Soc Robotics. 2016;8:157-81.

58. Koch S, Hägglund M. Health informatics and the delivery of care to older people. Maturitas. 2009;63:195-9.

59. Koceska N, Koceski S, Beomonte Zobel P, Trajkovik V, Garcia N. A telemedicine robot system for assisted and independent living. Sensors (14248220). 2019;19:834.

60. Chau CW, Leung E. Health informatics and technology for integrated elderly care in the context of Hong Kong: a case study. Stud Health Technol Inform. 2017;241:134-44.

61. Fischer SH, David D, Crotty BH, Dierks M, Safran C. Acceptance and use of health information technology by community-dwelling elders. Int J Med Inform. 2014;83:624-35

62. Bemelmans R, Gelderblom GJ, Jonker P, De Witte L. Socially assistive robots in elderly care: a systematic review into effects and effectiveness. J Am Med Dir Assoc. 2012;114.

63. Fausset CB, Kelly AJ, Rogers WA, Fisk AD. Challenges to aging in place: understanding home maintenance difficulties. J Hous Elderly. 2011;25:125-41.

64. Wang D, Subagdja B, Kang Y, Tan A-H, Zhang D. Towards intelligent caring agents for aging-in-place: issues and challenges. 2014 IEEE Symposium on Computational Intelligence for Human-like Intelligence (CIHLI). 2014. p. 1-8.

65. Ulrich RS, Zimring C, Zhu X, DuBose J, Seo H-B, Choi Y-S, et al. A review of the research literature on evidence-based healthcare design. HERD. 2008;1:61-125.

66. Agüero-Torres H, von Strauss E, Viitanen M, Winblad B, Fratiglioni L. Institutionalization in the elderly: the role of chronic diseases and dementia. Cross-sectional and longitudinal data from a population-based study. J Clin Epidemiol. 2001;54:795-801.

67. Chan J, Nejat G. Social intelligence for a robot engaging people in cognitive training activities. Int J Adv Robot Syst. 2012;9.

68. Majumder S, Aghayi E, Noferesti M, Memarzadeh-Tehran H, Mondal T, Pang Z, et al. Smart homes for elderly healthcarerecent advances and research challenges. Sensors (Basel). 2017;17. Available from: https://www.ncbi.nlm.nih.gov/pmc/ articles/PMC5712846/. Accessed 30 Dec 2019.

69. Sung H, Chang S, Chin M, Lee W. Robot-assisted therapy for improving social interactions and activity participation among institutionalized older adults: a pilot study. Asia Pac Psychiatry. 2015;7:1.
70. Cresswell K, Cunningham-Burley S, Sheikh A. Health Care Robotics: Qualitative Exploration of Key Challenges and Future Directions. J Med Internet Res. 2018;20. Available from: https:// www.ncbi.nlm.nih.gov/pmc/articles/PMC6053611/.

71. Fischinger D, Einramhof P, Papoutsakis K, Wohlkinger W, Mayer $\mathrm{P}$, Panek P, et al. Hobbit, a care robot supporting independent living at home: first prototype and lessons learned. Robot Auton Syst. 2016;75:60-78.

72. Coeckelbergh M. Health care, capabilities, and AI assistive technologies. Ethic Theory Moral Prac. 2010;13:181-90.

73. Coeckelbergh M, Pop C, Simut R, Peca A, Pintea S, David D, et al. A survey of expectations about the role of robots in robot-assisted therapy for children with ASD: ethical acceptability, trust, sociability, appearance, and attachment. Sci Eng Ethics. 2016;22:47-65.

74. Sparrow R, Sparrow L. In the hands of machines? The future of aged care. Mind Mach. 2006;16:141-61.

75. Iserson KV, Chiasson PM. The ethics of applying new medical technologies. Semin Laparosc Surg. 2002;9:222-9.

76. Norman ID, Aikins MK, Binka FN. Ethics and electronic health information technology: challenges for evidence-based medicine and the physician-patient relationship. Ghana Med J. 2011;45: $115-24$.

77. Feil-Seifer D, Matarić M. Ethical principles for socially assistive robotics. IEEE Robot Autom Mag. 2011;18:24-31.

78. Wada K, Shibata T, Musha T, Kimura S. Robot therapy for elders affected by dementia. IEEE Eng Med Biol Mag. 2008;27:53-60.

79. Powell-Cope G, Nelson AL, Patterson ES. Patient Care Technology and Safety. In: Hughes RG, editor. Patient Safety and Quality: An Evidence-Based Handbook for Nurses. Rockville: Agency for Healthcare Research and Quality (US); 2008. Available from: http://www.ncbi.nlm.nih.gov/books/NBK2686/. Accessed 30 Dec 2019.

80. Linner T, Pan W, Georgoulas C, Georgescu B, Güttler J, Bock T. Co-adaptation of robot systems, processes and in-house environments for professional care assistance in an ageing society. Procedia Eng. 2014;85:328-38.

81. Martinez-Martin E, Cazorla M. A socially assistive robot for elderly exercise promotion. IEEE Access. 2019;7:75515-29.

82. de Graaf MMA, Allouch SB, Klamer T. Sharing a life with Harvey: exploring the acceptance of and relationship-building with a social robot. Comput Hum Behav. 2015;43:1-14.

83. Wu Y-H, Wrobel J, Cornuet M, Kerhervé H, Damnée S, Rigaud A$\mathrm{S}$. Acceptance of an assistive robot in older adults: a mixed-method study of human-robot interaction over a 1-month period in the living lab setting. Clin Interv Aging. 2014;9:801-11.

84. All S, Nourbakhsh IR. Insect telepresence: using robotic teleembodiment to bring insects face-to-face with humans. Auton Robot. 2001;10:149-61.

85. Bakas T, Sampsel D, Israel J, Chamnikar A, Ellard A, Clark JG, et al. Satisfaction and technology evaluation of a telehealth robotic program to optimize healthy independent living for older adults. J Nurs Scholarsh. 2018;50:666-75.

86. Bevilacqua R, Cesta A, Cortellessa G, Macchione A, Orlandini A, Tiberio L. Telepresence robot at home: a long-term case study. 2014. p. 73-85.

87. Casiddu N, Cesta A, Cortellessa G, Orlandini A, Porfirione C, Divano A, et al. Robot Interface Design: The Giraff telepresence robot for social interaction. Biosystems and Biorobotics. 2015. p. 499-509.

Publisher's Note Springer Nature remains neutral with regard to jurisdictional claims in published maps and institutional affiliations. 